\title{
Long non-coding RNA: dancing on immune stage
}

\author{
HUANG Bo \\ State Key Laboratory of Medical Molecular Biology \& Department of Immunology, Institute of Basic Medical Sciences, Chinese Academy of \\ Medical Sciences \& Peking Union Medical College, Beijing 100005, China
}

Received April 23, 2014; accepted April 25, 2014; published online May 13, 2014

Citation: Huang B. Long non-coding RNA: dancing on immune stage. Sci China Life Sci, 2014, 57: 643-644, doi: 10.1007/s11427-014-4669-3

To date, molecular regulation of dendritic cell (DC) differentiation and DC-mediated immune deviation has been incompletely understood. DCs, which have long been recognized as highly potent antigen-presenting cells, play critical roles in linking innate immune functions with adaptive immunity. DCs belong to the hematopoietic system and arise from $\mathrm{CD} 34^{+}$stem cells in the bone marrow. After taking up antigenic materials, DCs process and present them to their membrane surface in the form of major histocompatibility complex (MHC) class I/or class II molecule-antigen peptide complexes, while concomitantly up-regulating costimulatory molecules and secreting a number of cytokines triggered by ambient inflammatory signaling. As a result, T cell activation is primed via an interaction between MHC-peptide complexes and $\mathrm{T}$ cell receptor (TCR), helped along by costimulatory signaling. It is the cytokines however that determine T cells biasing to develop toward Th1, Th2, Th17, cytotoxic T lymphocyte (CTL) or others. DCs are also involved in B lymphocyte maturation, Ig class-switching and antibody production. Beyond their antigen presentation function, DCs also play a critical role in tailoring immune response types and strength, mediating peripheral immune tolerance and maintaining immune homeostasis.

A new study by Wang et al. identifies a lncRNA expressed exclusively in human dendritic cells (DC), called lnc-DC, which is involved in DC differentiation and T cell priming [1]. The authors found that the knockdown of lnc-DC impaired DC differentiation from monocytes, in vitro, and in mouse bone marrow cells, in vivo, and reduced DC-induced $\mathrm{T}$ cell activation. An important transcription

email: tjhuangbo@hotmail.com factor, called signal transducer and activator of transcription 3 (STAT3), was found to be the target of lnc-DC. Lnc-DC promoted STAT3 phosphorylation on tyrosine-705 site by binding directly to STAT3 and subsequently preventing the binding of SHP1 to STAT3 for STAT3 dephosphorylation. The STAT proteins were originally recognized in the interferon system. A tyrosine kinase member called JAK is the primary activator of STATs. Other tyrosine kinases, such as Src and EGF receptor are also capable of activating STATs in certain given contexts. In line with this report, a previous study showed that STAT3 is a requirement of Flt3Ldependent DC differentiation [2]. However, in tumor models the activation of STAT3 may downregulate the expression of CD80, CD86 and IL-12 in DCs, leading to the inhibition of DC activation [3]. In knockout mice, STAT3 was demonstrated as a negative regulator of DC function [4]. The underlying molecular basis of this apparent inconsistence needs elucidation.

The regulation of STAT3 by long non-coding RNA (lncRNA) is intriguing. Unlike 19-23 nucleotide-length microRNA or other short RNAs, lncRNA is longer than 200 nucleotides and more abundant than small regulatory RNAs. LncRNAs exert their function through multiple ways: the process of transcribing lncRNAs can profoundly affect the ability of nearby genes to be expressed, due to its inhibiting RNA polymerase II recruitment or inducing chromatin remodeling; lncRNAs can also modulate the activity of protein-binding partners; and lncRNAs can be processed to yield small RNAs, such as miRNAs, piRNAs, and other small transcripts. Previous studies from the same lab have demonstrated that microRNAs such as miR-146 and miR-148/152 are involved in the regulation of DC functions 
and other immune cell subsets [5], suggesting non-coding RNAs play an important role in immune regulation. However, whether IncRNAs are important regulators of the immune system is poorly understood. The same group extended previous microRNA studies to the potential effect of lncRNA in immune regulation. By bioinformatic analysis and RNA sequencing (RNA-Seq), lnc-DC was screened and its action pathway was elegantly elucidated. It is believed that numerous long ncRNAs are misregulated in various illnesses. Whether abnormal expression of lnc-DC in DCs can be blamed for such diseases as cancer, infectious or autoimmune diseases is worthy of investigation. No doubt future study will provide many more unexpected insights into the functions of lnc-DC in physiology and pathophysiology.
1 Wang P, Xue Y, Han Y, Lin L, Wu C, Xu S, Jiang Z, Xu J, Liu Q, Cao $\mathrm{X}$. The STAT3-binding long noncoding RNA lnc-DC controls human dendritic cell differentiation. Science, 2014, 344: 310-313

2 Laouar Y, Welte T, Fu XY Flavell RA. STAT3 is required for Flt3L-dependent dendritic cell differentiation. Immunity, 2003, 19: 903-912

3 Kortylewski M, Kujawski M, Wang T, Wei S, Zhang S, Pilon-Thomas S, Niu G, Kay H, Mulé J, Kerr WG, Jove R, Pardoll D, Yu H. Inhibiting Stat3 signaling in the hematopoietic system elicits multicomponent antitumor immunity. Nat Med, 2005, 11: 1314-1321

4 Melillo JA, Song L, Bhagat G, Blazquez AB, Plumlee CR, Lee C, Berin C, Reizis B, Schindler C. Dendritic cell (DC)-specific targeting reveals Stat 3 as a negative regulator of DC function. J Immunol, 2010, 184: 2638-2645

5 Liu X, Zhan Z, Xu L, Ma F, Li D, Guo Z, Li N, Cao X. microRNA-148/152 impair innate response and antigen presentation of TLR-triggered dendritic cells by targeting CaMKII $\alpha$. J Immunol, 2010, 185: 7244-7251

Open Access This article is distributed under the terms of the Creative Commons Attribution License which permits any use, distribution, and reproduction in any medium, provided the original author(s) and source are credited. 